\title{
The three smallest compact arithmetic hyperbolic 5-orbifolds
}

\author{
VINCENT EMERY \\ RUTH KELLERHALS
}

\begin{abstract}
We determine the three hyperbolic 5-orbifolds of smallest volume among compact arithmetic orbifolds, and we identify their fundamental groups with hyperbolic Coxeter groups.
\end{abstract}

22E40; 11R42, 20F55, 51M25

In memoriam Friedrich Hirzebruch

\section{Introduction}

Let $\operatorname{Isom}\left(\mathbf{H}^{5}\right)$ be the group of isometries of the hyperbolic space $\mathbf{H}^{5}$ of dimension five, and Isom ${ }^{+}\left(\mathbf{H}^{5}\right)$ its index two subgroup of orientation-preserving isometries. In Belolipetsky and the first author's [1] (see also the first author's [5]) the lattice of smallest covolume among cocompact arithmetic lattices of $\operatorname{Isom}^{+}\left(\mathbf{H}^{5}\right)$ was determined. This lattice was constructed as the image of an arithmetic subgroup $\Gamma_{0}$ of the spinor group $\operatorname{Spin}(1,5)$ (note that $\operatorname{Spin}(1, n)$ is a twofold covering of $\operatorname{SO}(1, n)^{\circ} \cong \operatorname{Isom}^{+}\left(\mathbf{H}^{n}\right)$ ). More precisely, $\Gamma_{0}$ is given by the normalizer in $\operatorname{Spin}(1,5)$ of a certain arithmetic group $\Lambda_{0} \subset G_{0}\left(k_{0}\right)$, where $k_{0}=\boldsymbol{Q}(\sqrt{5})$ and $G_{0}$ is the algebraic $k_{0}$-group $\operatorname{Spin}\left(f_{0}\right)$ defined by the quadratic form

$$
f_{0}(x)=-(3+2 \sqrt{5}) x_{0}^{2}+x_{1}^{2}+\cdots+x_{5}^{2} .
$$

In [1], the index $\left[\Gamma_{0}: \Lambda_{0}\right]$ was computed to be equal to 2 . We note that it is easily checked that $\Lambda_{0}$ contains the center of $\operatorname{Spin}(1,5)$, so that the covolume of the action of $\Lambda_{0}$ on $\mathbf{H}^{5}$ is the double of the covolume of $\Gamma_{0}$.

In this article we construct a cocompact arithmetic lattice $\Gamma_{2} \subset \operatorname{Spin}(1,5)$ of covolume slightly bigger than the covolume of $\Lambda_{0}$, and we prove that it realizes the third smallest covolume among cocompact arithmetic lattices in $\operatorname{Spin}(1,5)$. In other words, we obtain the second and third values in the volume spectrum of compact orientable arithmetic hyperbolic 5-orbifolds, thus improving the results of [1] and [5] for this dimension. 


\begin{tabular}{cc} 
Lattice & Hyperbolic covolume \\
\hline$\Gamma_{0}^{\prime}$ & $0.00153459236 \ldots$ \\
$\Gamma_{1}^{\prime}$ & $0.00306918472 \ldots$ \\
$\Gamma_{2}^{\prime}$ & $0.00396939286 \ldots$ \\
& Table 1
\end{tabular}

\begin{tabular}{clc} 
Coxeter group & Coxeter symbol & Hyperbolic covolume \\
\hline$\Delta_{0}$ & {$[5,3,3,3,3]$} & $0.00076729618 \ldots$ \\
$\Delta_{1}$ & {$\left[5,3,3,3,3^{1,1}\right]$} & $0.00153459235 \ldots$ \\
$\Delta_{2}$ & {$[5,3,3,3,4]$} & $0.00198469643 \ldots$
\end{tabular}

Table 2: Approximation of hyperbolic covolumes

For notational reasons we put $\Gamma_{1}=\Lambda_{0}$. Moreover, for $i=0,1,2$, we denote by $\Gamma_{i}^{\prime}$ the image of $\Gamma_{i}$ in $\operatorname{Isom}^{+}\left(\mathbf{H}^{5}\right)$.

Theorem 1 The lattices $\Gamma_{0}^{\prime}, \Gamma_{1}^{\prime}$ and $\Gamma_{2}^{\prime}$ (ordered by increasing covolume) are the three cocompact arithmetic lattices in $\operatorname{Isom}^{+}\left(\mathbf{H}^{5}\right)$ of minimal covolume. They are unique, in the sense that any cocompact arithmetic lattice in $\operatorname{Isom}^{+}\left(\mathbf{H}^{5}\right)$ of covolume smaller than or equal to $\Gamma_{2}^{\prime}$ is conjugate in $\operatorname{Isom}\left(\mathbf{H}^{5}\right)$ to one of the $\Gamma_{i}^{\prime}$.

The precise formulas for the hyperbolic covolumes of these lattices are given below in Proposition 4. We list in Table 2 the corresponding numerical approximations.

A central motivation for Theorem 1 is that the lattices $\Gamma_{0}^{\prime}, \Gamma_{1}^{\prime}$ and $\Gamma_{2}^{\prime}$ can be related to concrete geometric objects. Namely, let $P_{0}$ and $P_{2}$ be the two compact Coxeter polytopes in $\mathbf{H}^{5}$ described by the following Coxeter diagrams, of respective Coxeter symbols $[5,3,3,3,3]$ and $[5,3,3,3,4]$ (see Section 4):

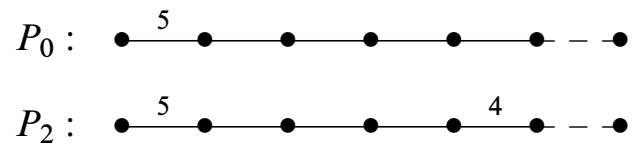

These two polytopes were first discovered by Makarov [10] (see also Im Hof [7] and Section 4). Combinatorially, they are simplicial prisms. Let $P_{1}=D P_{0}$ be the geometric double of $P_{0}$ with respect to its Coxeter facet $[5,3,3,3]$. It follows that the Coxeter polytope $P_{1}$ can be characterized by the following Coxeter diagram, of symbol $\left[5,3,3,3,3^{1,1}\right]$ :

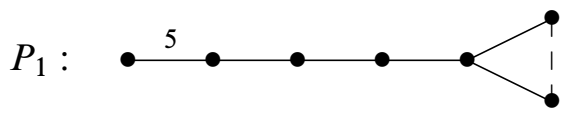


We denote by $\Delta_{i} \subset \operatorname{Isom}\left(\mathbf{H}^{5}\right)$ the Coxeter group generated by the reflections through the hyperplanes delimiting $P_{i}(0 \leq i \leq 2)$. It is known, by Vinberg's criterion [14], that the lattices $\Delta_{0}$ (thus $\Delta_{1}$ as well) and $\Delta_{2}$ are arithmetic.

Theorem 2 For $i=0,1,2$, let $\Delta_{i}^{+}$be the lattice $\Delta_{i} \cap \operatorname{Isom}^{+}\left(\mathbf{H}^{5}\right)$, which is of index two in $\Delta_{i}$. Then $\Delta_{i}^{+}$is conjugate to $\Gamma_{i}^{\prime}$ in $\operatorname{Isom}\left(\mathbf{H}^{5}\right)$. In particular, $\Delta_{0}$ realizes the smallest covolume among the cocompact arithmetic lattices in $\operatorname{Isom}\left(\mathbf{H}^{5}\right)$.

The proof of Theorem 2 is obtained as a consequence of Theorem 1 (more exactly from the slightly more precise Proposition 6) together with an geometric/analytic computation of the volumes $\operatorname{vol}\left(P_{0}\right)$ and $\operatorname{vol}\left(P_{2}\right)$ that will be presented in Section 4. We note that the fact that $\Delta_{2}$ and $\Gamma_{2}^{\prime}$ are commensurable lattices follows from the work of Bugaenko [3], where $\Delta_{2}$ is constructed by applying Vinberg's algorithm on the same quadratic form (2-1) which we will use below to construct $\Gamma_{2}$. No arithmetic construction of $\Delta_{0}$ and $\Delta_{1}$ was known so far.

The approximations of the volumes of $P_{0}, P_{1}$ and $P_{2}$ are listed in Table 2. These volumes can be obtained by two completely different approaches: from the method given in Section 4, or from the covolumes of the arithmetic lattices $\Gamma_{i}$, which are essentially computed with Prasad's volume formula [13]. The comparison of these two approaches has some arithmetic significance that will be briefly discussed in Section 5 .

Acknowledgements We would like to thank Herbert Gangl for interesting discussions concerning Section 5. We thank the Institut Mittag-Leffler in Stockholm, where this paper was completed. The first named author is thankful to the Max Planck Institute for Mathematics in Bonn for the hospitality and the financial support. The second named author was partially supported by the Swiss National Science Foundation, project number 200020-131967

\section{Construction and properties of $\Gamma_{2}$}

We call an algebraic group admissible if it gives rise to cocompact lattices in $\operatorname{Spin}(1,5)$; see [1, Section 2.2] for the exact definition. We say that an admissible $k$-group $G$ is associated with $\ell / k$, where $\ell$ is the smallest field extension of $k$ (necessarily quadratic) such that $G$ is an inner form over $\ell$, sometimes called "splitting field" of $G$. We use the same terminology for the arithmetic subgroups of $G$. Admissibility imposes that $G$ is of type ${ }^{2} \mathrm{~A}_{3}$, the field $k$ is totally real and $\ell$ has signature $(2, d-1)$, where $d=[k: \boldsymbol{Q}]$ (see [1, Proposition 2.5]). Note that since we consider only cocompact lattices in this article, we assume that $k \neq Q$. In the following, the symbol $V_{\mathrm{f}}$ will always refer to the set of finite places of the base field $k$ (and not of $\ell$ ). 
Let $G_{2}$ be the algebraic spinor group $\operatorname{Spin}\left(f_{2}\right)$ defined over $k_{0}=\boldsymbol{Q}(\sqrt{5})$, where $f_{2}$ is the following quadratic form:

$$
f_{2}(x)=-\omega x_{0}^{2}+x_{1}^{2}+\cdots+x_{5}^{2},
$$

with $\omega=(1+\sqrt{5}) / 2$. We have $G_{2}(\boldsymbol{R}) \cong \operatorname{Spin}(1,5) \times \operatorname{Spin}(6)$, proving that $G_{2}$ is admissible. Its "splitting field" is given by (see [1, Section 3.2]):

$$
\ell_{2}=\boldsymbol{Q}(\sqrt{\omega}) \cong \boldsymbol{Q}[x] /\left(x^{4}-x^{2}-1\right),
$$

which has a discriminant of absolute value $\mathscr{D}_{\ell_{2}}=400$. The following proposition shows an analogy between $G_{2}$ and $G_{0}$ (see [1, Proposition 3.6]).

Proposition 3 The group $G_{2}$ is quasisplit at every finite place $v$ of $k_{0}$. It is the unique admissible group associated with $k_{0} / \ell_{2}$ with this property.

Proof Since $\omega$ is an integer unit in $k_{0}$ it easily follows that for at each nondyadic place $v \neq(2)$ the form $f_{2}$ has the same Hasse symbol as the standard split form of signature $(3,3)$. From the structure theory of Spin described in [1, Section 3.2] we conclude that $G_{2}$ must be quasisplit at every finite place $v$ (note that at the place $v=(2)$, which is ramified in $\ell_{2} / k_{0}$, the group $G_{2}$ is necessarily an outer form). Similarly to the proof of [1, Proposition 3.6], the second affirmation follows from [1, Lemma 3.4] together with the Hasse-Minkowski Theorem.

We write here $k=k_{0}$. By Proposition 3 we see that for every finite place $v \in V_{\mathrm{f}}$ there exists a special parahoric subgroup $P_{v} \subset G_{2}\left(k_{v}\right)$. More precisely, $P_{v}$ is hyperspecial unless $v$ is the dyadic place (2) (the particularity of $v=(2)$ comes from the fact that this place is ramified in the extension $\left.\ell_{2} / k_{0}\right)$. The collection $\left(P_{v}\right)_{v \in V_{\mathrm{f}}}$ of special parahoric subgroups can be chosen to be coherent, ie such that $\prod_{v} P_{v}$ is open in the group $G_{2}\left(\boldsymbol{A}_{\mathrm{f}}\right)$ of finite adelic points. We now consider the principal arithmetic subgroup associated with such a coherent collection:

$$
\Lambda_{2}=G_{2}\left(k_{0}\right) \cap \prod_{v \in V_{\mathrm{f}}} P_{v} .
$$

The covolume of $\Lambda_{2}$ can be computed with Prasad's volume formula [13]. If $\mu$ denotes the Haar measure on $\operatorname{Spin}(1,5)$ normalized as in [1] (which corresponds to the measure $\mu_{\mathrm{S}}$ in [13]), then we obtain

$$
\mu\left(\Lambda_{2} \backslash \operatorname{Spin}(1,5)\right)=\mathscr{D}_{k_{0}}^{15 / 2} \mathscr{D}_{\ell_{2}}^{5 / 2} C^{2} \zeta_{k_{0}}(2) \zeta_{k_{0}}(4) L_{\ell_{2} / k_{0}}(3),
$$

where $C=3 \cdot 2^{-7} \pi^{-9}$, the symbol $\zeta_{k}$ denotes the Dedekind zeta function associated with $k$ and $L_{\ell / k}=\zeta_{\ell} / \zeta_{k}$ is the $L$-function corresponding to a quadratic extension $\ell / k$. 
We can now construct the group $\Gamma_{2}$ and compute its hyperbolic covolume. In the same proposition we recall the value of the hyperbolic covolume of $\Gamma_{0}$, which was obtained in [1].

Proposition 4 Let $\Gamma_{2}$ be the normalizer of $\Lambda_{2}$ in $\operatorname{Spin}(1,5)$. Then $\Lambda_{2}$ has index two in $\Gamma_{2}$. It follows that the hyperbolic covolume of $\Gamma_{2}^{\prime}$ is equal to

$$
\frac{9 \sqrt{5}^{15}}{2^{3} \pi^{15}} \zeta_{k_{0}}(2) \zeta_{k_{0}}(4) L_{\ell_{2} / k_{0}}(3)=0.00396939286 \ldots
$$

The hyperbolic covolume of $\Gamma_{0}^{\prime}$ is equal to

$$
\frac{9 \sqrt{5}^{15} \sqrt{11}^{5}}{2^{14} \pi^{15}} \zeta_{k_{0}}(2) \zeta_{k_{0}}(4) L_{\ell_{0} / k_{0}}(3)=0.00153459236 \ldots,
$$

where $\ell_{0}$ is the quartic field with $x^{4}-x^{3}+3 x-1$ as defining polynomial.

Proof The relation between the measure $\mu$ and the hyperbolic volume is described in [1, Section 2.1], where it is proved that in dimension 5 the hyperbolic covolume corresponds to the covolume with respect to $2 \pi^{3} \times \mu$. Thus it remains to prove that $\left[\Gamma_{2}: \Lambda_{2}\right]=2$. Let $k=k_{0}$.

It follows from the theory developed in $[1$, Section 4$]$ that the index $\left[\Gamma_{2}: \Lambda_{2}\right]$ is equal to the order of the group denoted by $A_{\xi}$ in [1], which can be identified as a subgroup of index at most two in $\mathbf{A}_{4} /\left(\ell_{2}^{\times}\right)^{4}$, where

$$
\mathbf{A}=\left\{x \in \ell_{2}^{\times} \mid N_{\ell_{2} / k}(x) \in\left(k^{\times}\right)^{4} \text { and } x>0\right\},
$$

$\mathbf{A}_{4}=\left\{x \in \mathbf{A} \mid v(x) \in 4 Z\right.$ for each normalized valuation $v$ of $\left.\ell_{2}\right\}$.

Note that in particular, for the integers $q$ and $q^{\prime}$ introduced in [1, Section 4.9], we have $q=\bar{q}=1$. Moreover, if $v=(2)$ denotes the (unique) ramified place of $\ell_{2} / k$, the subgroup $A_{\xi}$ is proper of index two in $\mathbf{A}_{4} /\left(\ell_{2}^{\times}\right)^{4}$ if and only if there exists an element of $\mathbf{A}_{4}$ acting nontrivially on the local Dynkin diagram $\Delta_{v}$ of $G_{2}\left(k_{v}\right)$. The action of $\mathbf{A}$ on $\Delta_{v}$ comes from its identification as a subgroup of the first Galois cohomology group $H^{1}(k, \mathrm{C})$ (where $\mathrm{C}$ is the center of $G_{2}$ ), which acts on every local Dynkin diagram associated with $G_{2}$. Since $G_{2}$ is of type A, we can use the results of [12, Section 4.2], which show that if $\pi_{w} \in \ell_{2}$ is a uniformizer for the ramified place $w \mid v$ of $\ell_{2}$, then $s=\pi_{w} \bar{\pi}_{w}^{-1}$ is a generator of the group $\operatorname{Aut}\left(\Delta_{v}\right)$. Taking $\pi_{w}=1+\omega+\sqrt{\omega}$, we obtain a positive unit $s$ acting nontrivially on $\Delta_{v}$. Thus, $A_{\xi}$ has index two in $\mathbf{A}_{4} /\left(\ell_{2}^{\times}\right)^{4}$. But the order of this latter group was computed in [1, Section 7.5] to be equal to 4 . This gives $\left[\Gamma_{2}: \Lambda_{2}\right]=2$. 
The "uniqueness" part of Theorem 1 requires the following result.

Proposition $5 U$ to conjugacy, the image of $\Gamma_{2}$ in $\operatorname{Isom}\left(\mathbf{H}^{5}\right)$ does not depend on the choice of a coherent collection of special parahoric subgroups $P_{v} \subset G_{2}\left(k_{v}\right)$.

Proof To prove this we can follow the same line of arguments as in [1, Section 6], where the result is proved for $\Gamma_{0} \subset G_{0}$ (our situation corresponding to the case of the type ${ }^{2} \mathrm{D}_{2 m+1}$ ). Thus, using [1, Section 6.5], the result follows by checking that $\mathbf{L} / \mathbf{A}$ and $U_{\mathbf{L}} / U_{\mathbf{A}}$ have the same order (equal to 2), where

$$
\mathbf{L}=\left\{x \in \ell_{2}^{\times} \mid N_{\ell_{2} / k_{0}}(x) \in\left(k_{0}^{\times}\right)^{4}\right\}
$$

and $U_{\mathbf{L}}$ (respectively $U_{\mathbf{A}}$ ) is the intersection of $\mathbf{L}$ (respectively $\mathbf{A}$ ) with the integers units in $\ell_{2}$.

\section{Proof of Theorem 1}

In view of Proposition 4, Theorem 1 is a direct consequence of the following statement.

Proposition 6 Let $\Gamma^{\prime} \subset \operatorname{Isom}^{+}\left(\mathbf{H}^{5}\right)$ be a cocompact arithmetic lattice that is not conjugate to $\Gamma_{0}^{\prime}, \Gamma_{1}^{\prime}$ or $\Gamma_{2}^{\prime}$. Then $\operatorname{vol}\left(\Gamma^{\prime} \backslash \mathbf{H}^{5}\right)>4 \cdot 10^{-3}$.

Proof Let $\Gamma \subset \operatorname{Spin}(1,5)$ be the full inverse image of $\Gamma^{\prime}$. We suppose that $\Gamma$ is an arithmetic subgroup of the group $G$, associated with $\ell / k$. From the values given in (2-5) and (2-6), it is clear that if $\Gamma$ is a proper subgroup of $\Gamma_{0}, \Gamma_{1}$ or $\Gamma_{2}$, then $\operatorname{vol}\left(\Gamma^{\prime} \backslash \mathbf{H}^{5}\right)>4 \cdot 10^{-3}$. Thus it suffices to prove the result assuming that $\Gamma$ is a maximal arithmetic subgroup with respect to inclusion. In particular, $\Gamma$ can be written as the normalizer of the principal arithmetic subgroup $\Lambda$ associated with some coherent collection $P=\left(P_{v}\right)$ of parahoric subgroups $P_{v} \subset G\left(k_{v}\right)$.

First we suppose that $k=k_{0}$ and $\ell=\ell_{0}$ or $\ell_{2}$. By Proposition 3 and its analogue for $G_{0}$, if $G$ is not isomorphic to $G_{0}$ or $G_{2}$ then at least one $P_{v}$ is not special. In particular, a "lambda factor" $\lambda_{v} \geq 18$ appears in the volume formula of $\Lambda$ [1, Section 7.1]. Together with [1, Equation (15)] (note that we do not assume here that $\Gamma=\Gamma^{\mathfrak{m}}$, in the notation of [1]) this shows that the covolume of $\Gamma$ is at least 9 times the covolume of $\Gamma_{0}$. Now if $G$ is isomorphic to $G_{0}$ or $G_{2}$, Proposition 5 and its analogue for $G_{0}$ show that at least one $P_{v}$ is not special, and the same argument as above applies.

Now we consider the situation $(k, \ell) \neq\left(k_{0}, \ell_{0}\right)$ nor $\left(k_{0}, \ell_{2}\right)$. We will use the different lower bounds for the covolume of $\Gamma$ given in [1, Section 7]. Note that in our case the rank $r$ of $G$ is equal to 3 . The notations are the following: $d$ is the degree of $k$, 
$\mathscr{D}_{k}$ and $\mathscr{D}_{\ell}$ are the discriminants of $k$ and $\ell$ in absolute value, and $h_{\ell}$ is the class number of $\ell$. Moreover, we set $a=3^{3} 2^{-4} \pi^{-11}$. From [1, Equation (37)] we have for $d \geq 7$ the following lower bound, which proves the result in this case (recall that the hyperbolic volume corresponds to $2 \pi^{3} \times \mu$, where $\mu$ is the Haar measure used by Prasad):

$$
\operatorname{vol}\left(\Gamma^{\prime} \backslash \mathbf{H}^{5}\right)>\frac{2 \pi^{3}}{32}\left(9.3^{5.5} \cdot a\right)^{7}=7.657 \ldots
$$

The following bound corresponds to [1, Equation (35)]:

$$
\operatorname{vol}\left(\Gamma^{\prime} \backslash \mathbf{H}^{5}\right)>\frac{2 \pi^{3}}{32} \mathscr{D}_{k}^{5.5} a^{d}
$$

For each degree $d=2, \ldots, 6$ we can use (3-2) to prove the result for a discriminant $\mathscr{D}_{k}$ high enough (eg $\mathscr{D}_{k} \geq 27$ for $d=2$ ). This leave us with a finite number of possible fields $k$ to examine. From these bounds on $\mathscr{D}_{k}$ and the tables of number fields (such as [2] and [6]) we obtain a list of nineteen fields $k$ (none of degree $d=6$ ) that remain to check.

Let us further consider the two following bounds, corresponding to [1, Equations (34) and (31)]. See (2-4) for the value of the symbol $C$ :

$$
\begin{aligned}
& \operatorname{vol}\left(\Gamma^{\prime} \backslash \mathbf{H}^{5}\right)>\frac{2 \pi^{3}}{32} \mathscr{D}_{k}^{2.5} \mathscr{D}_{\ell}^{1.5} a^{d}, \\
& \operatorname{vol}\left(\Gamma^{\prime} \backslash \mathbf{H}^{5}\right)>\frac{2 \pi^{3}}{h_{\ell} 2^{d+1}} \mathscr{D}_{k}^{7.5}\left(\mathscr{D}_{\ell} / \mathscr{D}_{k}^{2}\right)^{2.5} C^{d} .
\end{aligned}
$$

For each of the nineteen fields $k$ we easily obtain an upper bound $b_{k}$ for $\mathscr{D}_{\ell}$ for which the right hand side of (3-3) is at most $4 \cdot 10^{-3}$. Thus we only need to analyse the fields $\ell$ with $\mathscr{D}_{\ell} \leq b_{k}$. Let us fix a field $k$. The computational method described [4], based on class field theory, allows to determine all the quadratic extensions $\ell / k$ with $\mathscr{D}_{\ell} \leq b_{k}$ and with $\ell$ of the right signature, that is, $(2, d-1)$ (see [1, Proposition 2.5]). More precisely, we obtain this list of $\ell / k$ by programming a procedure in PARI/GP that uses the built-in functions bnrinit and rnfkummer. For each pair $(k, \ell)$ obtained, PARI/GP gives us the class number $h_{\ell}$ (checking its correctness with bnf certify) and this information makes (3-4) usable. The inequality $\operatorname{vol}\left(\Gamma^{\prime} \backslash \mathbf{H}^{5}\right)>4 \cdot 10^{-3}$ follows then for all the remaining $(k, \ell)$ except for the two situations:

$$
\begin{aligned}
& \left(\mathscr{D}_{k}, \mathscr{D}_{\ell}\right)=(8,448), \\
& \left(\mathscr{D}_{k}, \mathscr{D}_{\ell}\right)=(5,475) .
\end{aligned}
$$

The case (3-6) follows from Proposition 7 below. Let us then consider the case associated with (3-5). The smallest possible covolume of a maximal arithmetic subgroup 
$\Gamma=N_{\operatorname{Spin}(1,5)}(\Lambda)$ associated with $\ell / k$ would be in the situation when all parahoric subgroups $P_{v}$ determining $\Lambda$ are special. In this case, by [1, Proposition 4.12] the index $[\Gamma: \Lambda]$ is bounded by 8 , and together with the precise covolume of $\Lambda$ by Prasad's formula, we obtain (using PARI/GP to evaluate the zeta functions):

(3-7) $\operatorname{vol}\left(\Gamma^{\prime} \backslash \mathbf{H}^{5}\right) \geq \frac{2 \pi^{3}}{8} \mathscr{D}_{k}^{7.5}\left(\mathscr{D}_{\ell} / \mathscr{D}_{k}^{2}\right)^{2.5} C^{2} \zeta_{k}(2) \zeta_{k}(4) \zeta_{\ell}(3) / \zeta_{k}(3)=0.004997 \ldots$

This concludes the proof.

Proposition 7 Let $\ell$ be the quadratic extension of $k_{0}=\boldsymbol{Q}(\sqrt{5})$ with discriminant of absolute value $\mathscr{D}_{\ell}=475$. There exists a cocompact arithmetic lattice in $\operatorname{Isom}^{+}\left(\mathbf{H}^{5}\right)$ associated with $\ell / k_{0}$ whose approximate hyperbolic covolume is $0.006094 \ldots$ This is the smallest covolume among arithmetic lattices in $\operatorname{Isom}^{+}\left(\mathbf{H}^{5}\right)$ associated with $\ell / k_{0}$.

Proof Let $k=k_{0}$. The field $\ell$ can be concretely described as $\ell=k(\sqrt{\beta})$, where $\beta=-1+2 \sqrt{5}$ (this is a divisor of 19). We consider the algebraic group $G=\operatorname{Spin}(f)$ defined over $k=k_{0}$, with

$$
f(x)=-\beta x_{0}^{2}+x_{1}^{2}+\cdots+x_{5}^{2} .
$$

Similarly to [1, Proposition 3.6], we have that $G$ is quasisplit at every finite place $v \in V_{\mathrm{f}}$ (note that the proof for the unique dyadic place can be simplified in [1] by noting 2 is inert in $\ell$ and thus $G$ must be an outer form, necessarily quasisplit; see [1, Section 3.2]). It follows that there exist a coherent collection of special parahoric subgroups $P_{v} \subset G\left(k_{v}\right)$, and by Prasad's volume formula the hyperbolic covolume of an associated principal arithmetic subgroup $\Lambda$ is given by

$$
\operatorname{vol}\left(\Lambda \backslash \mathbf{H}^{5}\right)=2 \pi^{3} \mathscr{D}_{k}^{7.5}\left(\mathscr{D}_{\ell} / \mathscr{D}_{k}^{2}\right)^{2.5} C^{2} \zeta_{k}(2) \zeta_{k}(4) \zeta_{\ell}(3) / \zeta_{k}(3) .
$$

The index $[\Gamma: \Lambda]$ of $\Lambda$ in its normalizer $\Gamma$ can be computed using the same method as in the proof of Proposition 4. That the group $\mathbf{A}_{4} /\left(\ell^{\times}\right)^{4}$ has order 4 was already computed in [1, Section 7.5]. We use again [12, Section 4.2] to analyse the behavior at the ramified place $v=(\beta)$ : For the uniformizer $\pi_{w}=(\sqrt{\beta}+\beta) / 2$ of the place $w \mid v$ we get that $s=\pi_{w} \bar{\pi}_{w}^{-1}$ is an element of $\mathbf{A}_{4}$ that acts nontrivially on the local Dynkin diagram $\Delta_{v}$ of $G\left(k_{v}\right)$. As in the proof of Proposition 4 it follows that $[\Gamma: \Lambda]=2$. From (3-9) we obtain the value $0.006094 \ldots$ as the hyperbolic covolume of $\Gamma$. That no other arithmetic group associated with $\ell / k$ has smaller covolume follows from [1, Section 4.3] (since $\Lambda$ is of the form $\Lambda^{\mathfrak{m}}$; see [5, Section 12.3] for more details). 


\section{Proof of Theorem 2}

Consider the vector space model $\boldsymbol{R}^{1,5}$ for $\mathbf{H}^{5}$ as above and represent a hyperbolic hyperplane $H=e^{\perp}$ by means of a space-like unit vector $e \in \boldsymbol{R}^{1,5}$. A hyperbolic Coxeter polytope $P=\bigcap_{i \in I} H_{i}^{-}$is the intersection of finitely many half-spaces (whose normal unit vectors are directed outwards with respect to $P$ ) whose dihedral angles are submultiples of $\pi$. The group $\Delta$ generated by the reflections with respect to the hyperplanes $H_{i}, i \in I$, is a discrete subgroup of $\operatorname{Isom}\left(\mathbf{H}^{5}\right)$. If the cardinality of $I$ is small, a Coxeter polytope and its reflection group are best represented by the Coxeter diagram or by the Coxeter symbol. To each limiting hyperplane $H_{i}$ of a Coxeter polytope $P$ corresponds a node $i$ in the Coxeter diagram, and two nodes $i, j$ are connected by an edge of weight $p$ if the hyperplanes intersect under the (non-right) angle $\pi / p$. Notice that the weight 3 will always be omitted. If two hyperplanes are orthogonal, their nodes are not connected. If they admit a common perpendicular (of length $l$ ), their nodes are joined by a dashed edge (and the weight $l$ is usually omitted). We extend the diagram description to arbitrary convex hyperbolic polytopes and associate with the dihedral angle $\alpha=\angle\left(H_{i}, H_{j}\right)$ an edge with weight $\alpha$ connecting the nodes $i, j$. For the intermediate case of quasi-Coxeter polytopes whose dihedral angles are rational multiples $(p / q) \pi$ of $\pi$, the edge weight will be $q / p$. The Coxeter symbol is a bracketed expression encoding the form of the Coxeter diagram in an abbreviated way. For example, $[p, q, r]$ is associated with a linear Coxeter diagram with 3 edges of consecutive markings $p, q, r$. The Coxeter symbol $\left[3^{i, j, k}\right]$ denotes a group with Y-shaped Coxeter diagram with strings of $i, j$ and $k$ edges emanating from a common node. However, dashed edges are omitted leaving a connected graph. The Coxeter symbol can be extended to the quasi-Coxeter case in an obvious way as well.

\begin{tabular}{cccc} 
Group & Coxeter diagram & Coxeter symbol & Polytope \\
\hline$\Delta_{0}$ & $\bullet$ & $\bullet$ & $\bullet$
\end{tabular}

Table 3: Three hyperbolic Coxeter groups and their 5-polytopes

We are particularly interested in the quasi-Coxeter groups $\Delta_{i}$ and the polytopes $P_{i}$ (see Section 1) as given in Table 3. In order to compute the volumes of $P_{i}$, we consider the 1-parameter sequence of compact 5-prisms with symbol

$P(\alpha): \quad[5,3,3,3, \alpha]$, 
where $\alpha \in[\pi / 4,2 \pi / 5]$. Geometrically, they are compactifications of 5-dimensional orthoschemes obtained by cutting away the ultraideal principal vertices by the associated polar hyperplanes. The sequence (4-1) contains the Coxeter polytopes $P_{0}=[5,3,3,3,3]$ and $P_{2}=[5,3,3,3,4]$ as well as the pseudo-Coxeter prism [5, 3, 3, 3, 5/2]. There is no closed volume formula for such polytopes known in terms of the dihedral angles. However, for certain noncompact limiting cases and by means of scissors congruence techniques, exact volume expressions could be derived [8, Section 4.2]. For example,

$$
\begin{aligned}
\operatorname{vol}_{5}([5 / 2,3,3,5,5 / 2]) & =\frac{13 \zeta(3)}{9600}+\frac{11}{1152} J_{3}\left(\frac{\pi}{5}\right), \\
\operatorname{vol}_{5}([5,3,3,5 / 2,5]) & =-\frac{\zeta(3)}{4800}+\frac{11}{1152} J_{3}\left(\frac{\pi}{5}\right),
\end{aligned}
$$

and finally,

(4-4) $\operatorname{vol}_{5}(P(2 \pi / 5))=\frac{1}{5}\left(\operatorname{vol}_{5}([5 / 2,3,3,5,5 / 2])-\operatorname{vol}_{5}([5,3,3,5 / 2,5])\right)=\frac{\zeta(3)}{3200}$.

Here

$$
J_{3}(\omega)=\frac{1}{4} \sum_{r=1}^{\infty} \frac{\cos (2 r \omega)}{r^{3}}=\frac{1}{4} \zeta(3)-\int_{0}^{\omega} \pi_{2}(t) d t, \quad \omega \in \boldsymbol{R},
$$

denotes the Lobachevsky trilogarithm function, which is related to the real part of the classical polylogarithm $\operatorname{Li}_{k}(z)=\sum_{r=1}^{\infty} z^{r} / r^{k}$ for $k=3$ and $z=\exp (2 i \omega)$ (see [8, Section 4.1] and (4-10)).

For the volume calculation of the prisms $P_{0}$ and $P_{2}$, we apply the volume differential formula of L Schläfli (see [11], for example) with the reference value (4-4) in order to obtain the simple integral expression

$$
\operatorname{vol}_{5}(P(\alpha))=\frac{1}{4} \int_{\alpha}^{2 \pi / 5} \operatorname{vol}_{3}([5,3, \beta(t)]) d t+\frac{\zeta(3)}{3200},
$$

with a compact tetrahedron $[5,3, \beta(t)]$ whose angle parameter $\beta(t) \in] 0, \pi / 2[$ is given by

$$
\beta(t)=\arctan \sqrt{2-\cot ^{2} t} .
$$

Put

$$
\left.\theta(t)=\arctan \frac{\sqrt{1-4 \sin ^{2} \frac{\pi}{5} \sin ^{2} \beta(t)}}{2 \cos \frac{\pi}{5} \cos \beta(t)} \in\right] 0, \frac{\pi}{2}[.
$$


Then the volume of the 3-dimensional orthoscheme face $[5,3, \beta(t)]$ as given by Lobachevsky's formula (see [8, Equation (67)], for example) equals

$$
\begin{aligned}
\operatorname{vol}_{3}([5,3, \beta(t)])=\frac{1}{4}\left\{J_{2}\left(\frac{\pi}{5}+\theta(t)\right)\right. & -\pi_{2}\left(\frac{\pi}{5}-\theta(t)\right)-\pi_{2}\left(\frac{\pi}{6}+\theta(t)\right) \\
& +J_{2}\left(\frac{\pi}{6}-\theta(t)\right)+J_{2}(\beta(t)+\theta(t)) \\
& \left.-J_{2}(\beta(t)-\theta(t))+2 \Omega_{2}\left(\frac{\pi}{2}-\theta(t)\right)\right\},
\end{aligned}
$$

where

$$
J_{2}(\omega)=\frac{1}{2} \sum_{r=1}^{\infty} \frac{\sin (2 r \omega)}{r^{2}}=-\int_{0}^{\omega} \log |2 \sin t| d t, \quad \omega \in \boldsymbol{R},
$$

is Lobachevsky's function (in a slightly modified way).

The numerical approximation of the volumes of $P_{0}$ and $P_{2}$ can now be performed by implementing the data (4-7), (4-8) and (4-9) into the expression (4-6). We obtain, using the functions intnum and polylog in PARI/GP, that the three volumes of $P_{0}$, $P_{1}$ and $P_{2}$ (in increasing order) are clearly less than $2 \cdot 10^{-3}$. Since the groups $\Delta_{i}$ $(i=0,1,2)$ are known to be arithmetic, it follows then from Proposition 6 that their subgroups of index two $\Delta_{i}^{+}$must coincide with the $\Gamma_{i}^{\prime}$. This concludes the proof of Theorem 2.

\section{Remarks on the identification of volumes}

Although in the proof of Theorem 2 it suffices to use the rough estimate $\operatorname{vol}\left(P_{2}\right)<$ $2 \cdot 10^{-3}$, the numerical approximations are much more precise. Namely, the equality $\operatorname{vol}\left(\Gamma_{i}^{\prime} \backslash \mathbf{H}^{5}\right)=\operatorname{vol}\left(\Delta_{i}^{+} \backslash \mathbf{H}^{5}\right)$, proved by Theorem 2, yields for $i=0,2$ :

$$
\left\{\begin{aligned}
\frac{9 \sqrt{5}^{15} \sqrt{11}^{5}}{2^{14} \pi^{15}} \zeta_{k_{0}}(2) \zeta_{k_{0}}(4) L_{\ell_{0} / k_{0}}(3) & =2 \operatorname{vol}_{5}(P(\pi / 3)), \\
\frac{9 \sqrt{5}^{15}}{2^{3} \pi^{15}} \zeta_{k_{0}}(2) \zeta_{k_{0}}(4) L_{\ell_{2} / k_{0}}(3) & =2 \operatorname{vol}_{5}(P(\pi / 5)) .
\end{aligned}\right.
$$

Using PARI/GP, a computer checks within seconds that both sides of each equation coincide up to 50 digits (the right hand side being computed from (4-6) like in last step of Section 4).

The equalities (5-1) have also some arithmetic interest, due the presence on the left hand side of the special value $L_{\ell / k_{0}}$ (3) (with $\ell=\ell_{0}$ or $\ell_{2}$ ). Since $k_{0}$ is totally real, it follows from the Klingen-Siegel theorem (see [9]; see also [12, Appendix C]) that 
$\zeta_{k_{0}}(2) \zeta_{k_{0}}(4)$ is up to a rational given by some power of $\pi$ divided by $\sqrt{\mathscr{D}_{k_{0}}}=\sqrt{5}$. Thus, from (5-1) the nontrivial part $L_{\ell / k_{0}}$ (3) of $\operatorname{vol}\left(\Gamma_{i}^{\prime} \backslash \mathbf{H}^{5}\right)$ can be expressed by a sum of integrals of Lobachevsky's functions. A related but much more significant idea is the possibility, predicted by Zagier's conjecture, of expressing $L_{\ell / k_{0}}(3)$ as a sum of trilogarithms evaluated at integers of $k_{0}$. We refer to [15] for more information on this subject.

\section{References}

[1] M Belolipetsky, V Emery, On volumes of arithmetic quotients of $\mathrm{PO}(n, 1)^{\circ}, n$ odd, Proc. Lond. Math. Soc. 105 (2012) 541-570 MR2974199

[2] The Bordeaux database Available at ftp://megrez.math.u-bordeaux.fr/pub/ numberfields

[3] V O Bugaenko, Groups of automorphisms of unimodular hyperbolic quadratic forms over the ring $\mathbf{Z}[(\sqrt{5}+1) / 2]$, Vestnik Moskov. Univ. Ser. I Mat. Mekh. (1984) 6-12 MR764026 In Russian; translated in Moscow Univ. Math. Bull. 39:5 (1984) 6-14

[4] H Cohen, F Diaz y Diaz, M Olivier, Computing ray class groups, conductors and discriminants, Math. Comp. 67 (1998) 773-795 MR1443117

[5] V Emery, Du volume des quotients arithmétiques de l'espace hyperbolique, $\mathrm{PhD}$ thesis, University of Fribourg (2009)

[6] S Freundt, QaoS online database Available at http://qaos.math.tu-berlin.de

[7] H-C Im Hof, Napier cycles and hyperbolic Coxeter groups, Bull. Soc. Math. Belg. Sér. A 42 (1990) 523-545 MR1316209

[8] R Kellerhals, Scissors congruence, the golden ratio and volumes in hyperbolic 5-space, Discrete Comput. Geom. 47 (2012) 629-658 MR2891254

[9] H Klingen, Über die Werte der Dedekindschen Zetafunktion, Math. Ann. 145 (1961/1962) 265-272 MR0133304

[10] VS Makarov, The Fedorov groups of four-dimensional and five-dimensional Lobačevskiu space, from: "Studies in General Algebra”, volume 1, Kišinev. Gos. Univ., Kishinev (1968) 120-129 MR0259735

[11] J Milnor, The Schläfli differential equality, from: "Collected papers", volume 1, Publish or Perish, Houston, TX (1994) x+295 MR1277810

[12] A Mohammadi, A Salehi Golsefidy, Discrete subgroups acting transitively on vertices of a Bruhat-Tits building, Duke Math. J. 161 (2012) 483-544 MR2881229

[13] G Prasad, Volumes of $S$-arithmetic quotients of semi-simple groups, Inst. Hautes Études Sci. Publ. Math. (1989) 91-117 MR1019962 
[14] È B Vinberg, Discrete groups generated by reflections in Lobačevskĭ spaces, Math. USSR Sb. 1 (1967) 429-444 MR0207853

[15] D Zagier, H Gangl, Classical and elliptic polylogarithms and special values of $L-$ series, from: "The arithmetic and geometry of algebraic cycles", (B B Gordon, J D Lewis, S Müller-Stach, S Saito, N Yui, editors), NATO Sci. Ser. C Math. Phys. Sci. 548, Kluwer Acad. Publ., Dordrecht (2000) 561-615 MR1744961

Max Planck Institute for Mathematics

Vivatsgasse 7, D-53111 Bonn, Germany

Department of Mathematics, University of Fribourg

Chemin du Musée 23, CH-1700 Fribourg, Switzerland

vincent.emery@gmail.com, ruth.kellerhals@unifr.ch

Received: 24 August 2012 